\title{
Uranium resonance ionization mass spectrometry in natural U-silicate
}

\author{
By K. B. Knight ${ }^{1}$, , M. R. Savina' ${ }^{2}$, B. H. Isselhardt ${ }^{3}$, I. D. Hutcheon ${ }^{1}$, S. G. Prussin ${ }^{3}$ and M. J. Pellin ${ }^{2}$ \\ ${ }^{1}$ Lawrence Livermore National Laboratory, Livermore, CA, USA \\ 2 Argonne National Laboratory, USA \\ 3 The University of California, Berkeley, USA
}

(Received February 1, 2010; accepted in final form May 25, 2010)

Resonance ionization / Uranium / Mass spectrometry / RIMS / Isotope / Useful yield

Summary. A natural uranium silicate mineral, cuprosklodowskite, has been analyzed by Resonance Ionization Mass Spectrometry (RIMS) for uranium isotope composition under a variety of experimental conditions. While no significant isobaric interferences are observed in the mass regions of interest, molecular supression is incomplete, and varies with the method of neutral U atom production. Despite low measured useful yields under the present analytical arrangement, it is possible to measure uranium isotope composition directly from the sample surface with only a minimum of sample preparation. U-RIMS is a method deserving further development for analyses of environmental materials.

\section{Introduction}

Resonance ionization mass spectrometry (RIMS) is capable of isotopic analysis of debris and environmental material without sample purification; that is, material can be collected, placed directly into the instrument, imaged, and analyzed. Unlike most other mass spectrometric techniques, RIMS is also capable of isotopic analysis in the presence of isobaric interferences, and is generally insensitive to matrixdependent fractionation $[1,2]$. At the heart of the method is a suite of tunable lasers used to resonantly excite and ionize only one particular element from a complex sample made up of many different elements. Where other methods generally rely on dissolution chemistry to separate the element of interest from other species in a sample, RIMS does this chemical separation spectroscopically, inside the instrument. Thus, even complex materials can be analyzed directly, without the need for prior preparation. Robust measurement of uranium isotopes by RIMS using pulsed solid state lasers has recently been demonstrated on U-oxide standards with minimal sample preparation [3]. We applied similar methods to determine if direct measurement of uranium isotopes was applicable to more environmentally relevant samples. We present the first direct analyses of $\mathrm{U}$ isotopes by RIMS in

\footnotetext{
*Author for correspondence (E-mail: knight29@mail.1lnl.gov).
}

a complex natural silicate, including isotope ratios, a preliminary measurement of the useful yield, and a discussion of non-resonant ionization of uranium oxides.

RIMS has been applied to problems in analytical radiochemistry [2,4]. In RIMS, a mixture of neutral atoms, ions, and molecules is liberated from a sample surface by laser desorption or ion sputtering. A laser beam, with photons tuned to an energy corresponding to a specific electronic transition in the element of interest, is then directed through the plume of desorbed material, exciting neutral atoms of the element of interest into an intermediate state. A second and sometimes third laser beam, with a total photon energy sufficient to exceed the ionization threshold (often tuned to an autoionizing state above the ionization potential), is then passed through the cloud. This results in the ionization of only those atoms in the excited intermediate state, leaving other atomic species as neutral atoms. The selectively formed ions are subsequently accelerated and detected by conventional mass spectrometry. Because resonance ionization mass spectrometry does elemental separation spectroscopically inside the instrument, there is little need for chemical separation treatment prior to analysis.

One of the greatest challenges to attaining high precision in uranium isotope measurements by RIMS is the magnitude of the uranium isotope shift. Isotope shifts are caused by differences in the nuclear mass and/or nuclear volume of the isotopes of an element, and manifest as slight differences in the energies of the electronic states of the various isotopes. For example in the uranium $J=7$ state at $24066 \mathrm{~cm}^{-1}$ used in this work, the energies of ${ }^{235} \mathrm{U}$ and ${ }^{238} \mathrm{U}$ differ by $0.418 \mathrm{~cm}^{-1}$, corresponding to a difference in the resonance laser tuning of $\sim 7 \mathrm{pm}$. Since laser bandwidths are often much less than this, small, picometer-scale shifts in the wavelength of the resonance lasers can result in the preferential ionization of one isotope over another and cause large (orders of magnitude) shifts in measured uranium isotope ratios. Indeed, some RIMS applications use narrowband lasers specifically to achieve preferential ionization of single uranium isotopes $[5,6]$. In the experiments described here, we apply broadened resonance laser bandwidths centered on the resonances in different isotopes to minimize the isotopic fractionation due to the isotope shift (see Analytical Methods section). 
Recent work to enhance laser wavelength control and reduce the effect of isotope shifts has resulted in improved precision in isotope ratio measurements by RIMS, as demonstrated on uranium oxide isotopic standards [3]. Application to natural and low-level uranium-bearing samples, however, introduces background effects primarily from unsuppressed secondary ions as well as from multiphoton single wavelength ionization and fragmentation of molecules. This paper describes a preliminary study of a resonance ionization analytical method for the determination of uranium isotope ratios directly from a complex silicatedominated matrix with minimal sample preparation. The natural U-silicate, cuprosklodowskite, was chosen as an analog for environmental samples as well as debris from nuclear fallout.

\section{Analytical methods}

For these experiments we utilized the natural U-ore mineral cuprosklodowskite, a hydrated copper uranyl silicate with the mineral formula $\left(\mathrm{H}_{3} \mathrm{O}\right)_{2} \mathrm{Cu}\left(\mathrm{UO}_{2}\right)_{2}\left(\mathrm{SiO}_{4}\right)_{2} \cdot 2\left(\mathrm{H}_{2} \mathrm{O}\right)$. Fragments of cuprosklodowskite from a hand specimen (Musonoi Mine, Democratic Republic of the Congo) were mounted in resin on a $6 \mathrm{~mm} \mathrm{Al} \mathrm{disk,} \mathrm{polished} \mathrm{flat,} \mathrm{and} \mathrm{car-}$ bon coated to enhance conductivity (Fig. 1). The sample was imaged using backscattered scanning electron (BSE) microscopy and energy dispersive X-ray spectroscopy (EDS) to characterize mineral heterogeneity. We observed nearly pure fragments of cuprosklodowskite, which are the focus of the analyses presented here, as well as areas dominated by

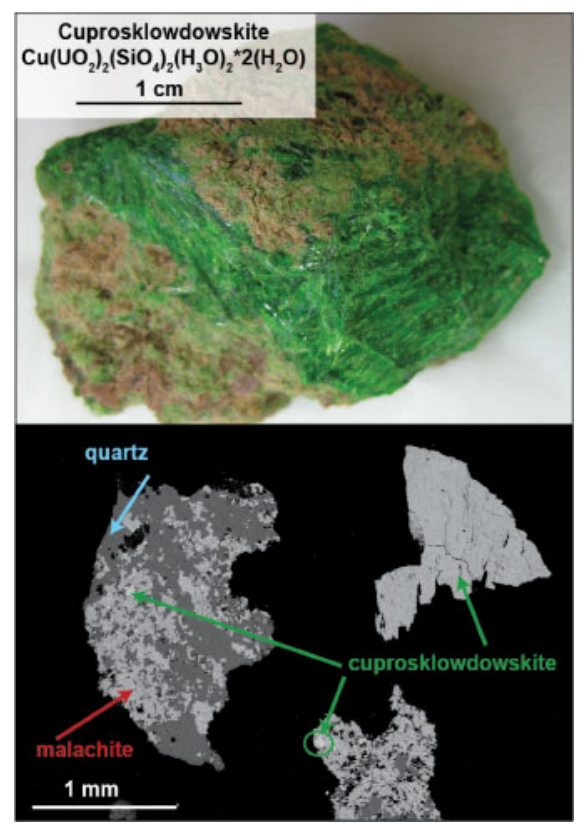

Fig. 1. Top: the U-silicate hand specimen showing the characteristic green color of cuprosklodowskite and the macro-scale fibrous habit. Bottom: a back-scattered electron image of the polished fragments mounted for this study. The mineralogical complexity is apparent. The darkest grey phase is quartz, while the intermediate grey phase is $\mathrm{Cu}-$ oxide. The lightest grey phase is U-bearing cuprosklodowskite. The nearly pure cuprosklodowskite fragment at the top right of the image was the focus of the present study. Sub-micron sized bright areas indicate minor Pb-rich sulfides.
$\mathrm{Cu}$-oxide (likely malachite), quartz and a minor $\mathrm{Pb}$-sulfide phase. The isotopic composition of the cuprosklodowskite was independently verified by ICP-MS to be ${ }^{235} \mathrm{U} /{ }^{238} \mathrm{U}=$ $0.00728 \pm 0.00004(2 \sigma)$, with a uranium concentration of $23.4 \%$ by weight.

Resonance ionization experiments were performed using the CHARIMSA instrument with Ti:Sapphire tunable lasers at Argonne National Laboratory [7]. CHARISMA is a timeof-flight mass spectrometer originally built and designed for RIMS analyses of interstellar dust grains recovered from meteorites. These dust grains tend to be small $(\sim 1 \mu \mathrm{m})$, but contain large isotopic anomalies. Such analyses require the high elemental selectivity and sensitivity of RIMS, but do not impose stringent requirements on precision. More recent adaptations of RIMS for isotope analyses require considerable improvement in the achievable precision, ultimately derived from control of the wavelength, power and positioning of the lasers (e.g., $[3,8])$.

Both 2-color (after [9]) and 3-color resonance ionization schemes (after [10]) were used in this work. Both schemes are shown in Fig. 2. Several intermediate uranium transitions can be excited by wavelengths within the range of the tunable Ti:Sapphire lasers employed on CHARISMA $(\sim 700-1000 \mathrm{~nm}$, and their doubled and tripled frequencies). The 2-color scheme utilized two frequency-doubled beams with $\sim 100 \mathrm{~mW}$ in the first laser and $250-300 \mathrm{~mW}$ in the second laser. The 3-color scheme used a frequency doubled first resonance laser set to a power of $80 \mathrm{~mW}$, and two $600-650 \mathrm{~mW}$ fundamental beams for the second resonance and ionization lasers. The resonance lasers for both the 2and 3-color schemes were tuned to the midpoint of the isotope splitting of ${ }^{238} \mathrm{U}$ and ${ }^{235} \mathrm{U}$ at the resonance of interest, as shown in Fig. 2.

To improve analytical precision, an automated feedback system was used to track and correct picometer-scale drift in the pulsed Ti:Sapphire laser wavelengths over the duration of each experiment. This system couples wavelength measurements with adjustments with picometer resolution to a holographic grating set on a moving stage in each Ti:Sapphire cavity (see [8] for details). In addition to controlling wavelength drift, we broadened the resonance laser bandwidth to $5-10 \mathrm{pm}$ by changing the anamorphic prism pair beam expanders which set the bandwidth in the Ti:Sapphire laser cavity. The broadened resonance laser bandwidth damped the variable isotopic fractionation that arises from pulse-to-pulse variations of the laser wavelength.

These experiments used a pulsed $25 \mathrm{keV}$ Ionoptika $\mathrm{Ga}^{+}$ ion gun to sputter atoms and molecules from the sample. The $\mathrm{Ga}^{+}$beam spot size was $\sim 4 \mu \mathrm{m}$, rastered over a $15-20 \mu \mathrm{m}$ area. We also performed several experiments utilizing a $3^{r d}$ harmonic Nd:YAG desorption laser $(355 \mathrm{~nm})$ to desorb material. The desorption laser beam spot was $\sim 2 \mu \mathrm{m}$ in diameter, and was also rastered over a 15-20 $\mu \mathrm{m}$ area. Secondary ions formed by interaction of the $\mathrm{Ga}^{+}$beam or desorption laser beam with the sample surface were suppressed by application of a brief positive voltage pulse $(+4000 \mathrm{~V})$ to the sample after the $\mathrm{Ga}^{+}$or desorption laser pulse, but before arrival of the resonance and ionization laser photons. Resonance and ionization lasers were timed to pulse simultaneously, $\sim 150 \mathrm{~ns}$ after the $\mathrm{Ga}^{+}$or desorption laser, creating near instantaneous formation of $\mathrm{U}$ ions. The target 


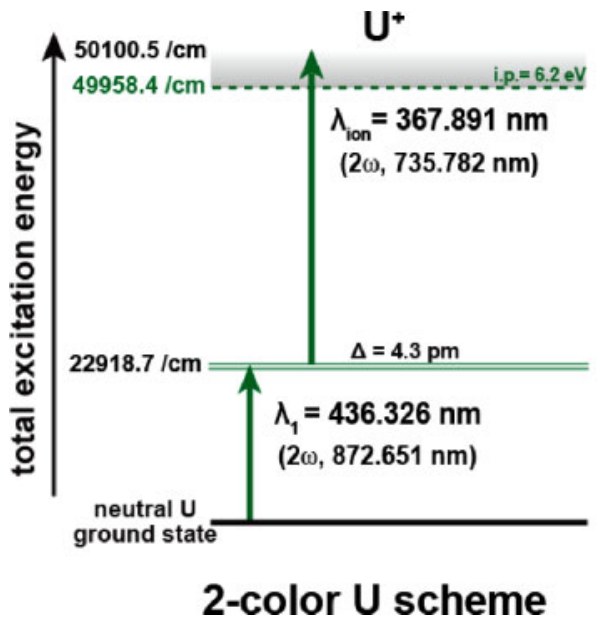

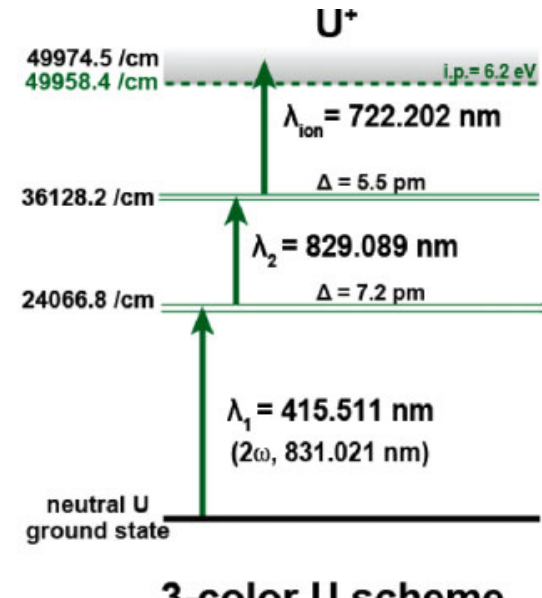

3-color U scheme
Fig. 2. The 2-color color [9] and 3color [10] uranium resonance ionization schemes used in this study. Broadened resonance laser bandwidths were used to encompass both peak excitation energies of the two uranium isotopes of interest, ${ }^{235} \mathrm{U}$ and ${ }^{238} \mathrm{U}$. and extraction optics were then pulsed to positive voltages $(+2000 \mathrm{~V}$ and $+1750 \mathrm{~V}$, respectively) to accelerate and focus the ions into a reflectron-type time-of-flight mass spectrometer. The onset of the target pulse acted as time zero in the time-of-flight system. The repetition rate of the entire system was $1 \mathrm{kHz}$.

Each individual ion impact on the microchannel plate detector was recorded as a function of time, creating characteristic mass spectra. An in-line discriminator removed signals from ion impacts that were less than $25 \mathrm{mV}$ in magnitude. This discriminates against electronic noise, but also removes $\sim 10 \%$ of the total signal. For efficient data collection and processing, $10^{5}$ laser pulses were analyzed as a single record. The number of records relevant to each of the following experiments is noted in Table 1. Total ion counts for a given isotope are derived from the integrated number of counts in the peaks and were corrected for deadtime using an empirical model derived from [11], but specific to the CHARISMA instrument. The magnitude of deadtime corrections for these experiments is less than $1 \%$ for the ${ }^{235} \mathrm{U} /{ }^{238} \mathrm{U}$ ratio. Reported ratios (Table 1) are not corrected for non-resonant contributions.

For this study, precision is a function of counting statistics, only. The presence of the large $U$ isotope shift, in par- ticular, makes reproducibility of the resonance and ionization wavelengths a primary source of fractionation between experiments. In light of this, correspondence of the measured ${ }^{235} \mathrm{U} /{ }^{238} \mathrm{U}$ ratio in the cuproskodowskite sample to the natural ${ }^{235} \mathrm{U} /{ }^{238} \mathrm{U}$ ratio is largely a reflection of the accuracy and reproducibility of the resonance and ionization wavelengths used in the experiments. Analytical reproducibility can be evaluated by use of a running standard, similar to the sample-standard bracketing applied in secondary ion mass spectrometery, but was not done in these preliminary experiments.

A total of seven different experimental configurations were chosen (Table 1). For the 3-color RIMS scheme (Fig. 2) we measured the isotopic composition of cuprosklodowskite using $\mathrm{Ga}^{+}$sputtering, and compared these data with detuned (off-resonance) spectra, as well as with spectra generated using laser desorption. Using the 2-color RIMS scheme and $\mathrm{Ga}^{+}$sputtering we measured the isotopic composition as well as the contributions of each of several independent ionization processes: the $\mathrm{Ga}^{+}$secondary ion contribution, and the contributions due to the resonance and ionization lasers acting independently of one another. As these experiments have variable durations, data are shown normalized to counts per $10^{6}$ laser shots.

Table 1. Uranium RIMS experiments ${ }^{a}$.

\begin{tabular}{lrrrrrrr}
\hline Experiment & \# of runs & ${ }^{235} \mathrm{U}$ cts & ${ }^{238} \mathrm{U}$ cts & ${ }^{235} \mathrm{U} /{ }^{238} \mathrm{U}$ & $1 \sigma$ & $\mathrm{X}^{2}$ & $\mathrm{U}_{/} \mathrm{UO}_{x}$ \\
\hline 3-color & & & & & & & \\
$\mathrm{Ga}^{+}$sputtering on cuprosklodowskite & 9 & 94 & 14301 & 0.00657 & 0.00020 & 1.5 & 0.035 \\
laser desorption on cuprosklodowskite & 5 & 64 & 8488 & 0.00756 & 0.00044 & 0.9 & 0.417 \\
$\mathrm{Ga}^{+}$sputtering, off-resonance tuning & 1 & 12 & 680 & & & & 0.002 \\
2-color & 14 & 545 & 76110 & 0.00727 & 0.00008 & 1.4 & 0.055 \\
$\mathrm{Ga}^{+}$sputtering on cuprosklodowskite & 1 & 8 & 9 & & & & 0.018 \\
$\mathrm{Ga}^{+}$sputtering, only & 1 & 18 & 533 & & & 0.002 \\
$\mathrm{Ga}^{+}$sputtering and resonance beam only & 1 & 150 & 21347 & & & & 0.017 \\
$\mathrm{Ga}^{+}$sputtering and ionization beam only & 1 & & & & & & \\
\hline
\end{tabular}

a: Total integrated counts in the peaks are corrected for deadtime and normalized to the total number of runs. Each run consisted of 100000 laser shots. $1 \sigma$ errors are based on counting statistics, only. $\mathrm{UO}_{x} / \mathrm{U}$ is the sum of all $\mathrm{UO}$ and $\mathrm{UO}_{2}$ counts divided by the total number of $\mathrm{U}$ counts. Variation in the measured ${ }^{235} \mathrm{U} /{ }^{238} \mathrm{U}$ ratio by RIMS to the independently determined (natural) ${ }^{235} \mathrm{U} /{ }^{238} \mathrm{U}$ ratio is dominated by spectroscopic fractionation caused by inaccurate laser wavelength positioning. To best capture the precision and in-run variations, reported ${ }^{235} \mathrm{U} /{ }^{238} \mathrm{U}$ ratios represent the average and standard deviation based on the individual run data, and thus may vary slightly from the ratio one would derive from a simple ratio of the total number of counts of each isotope. 


\section{3-color RIMS in U-silicates}

Three 3-color experiments were performed on cuprosklodowskite. The first used $\mathrm{Ga}^{+}$sputtering, applying $\sim 10 \mathrm{nA}$ of current to the sample surface and collecting data over $9 \times 10^{5}$ laser shots. An average of $1.4 \times 10^{4}{ }^{238} \mathrm{U}$ ions per $10^{5}$ laser shots was observed with no significant off-mass background present in the U mass region (Fig. 3). Uranium oxides, however, were abundant, dominated by ${ }^{238} \mathrm{UO}$ at mass 254 and ${ }^{238} \mathrm{UO}_{2}$ at mass 270 . The observed $\mathrm{U}$ atom-tooxide ratio was 0.035 , with the number skewed slightly due to undercounting of the signal from the large oxide peaks.

A second experiment used the same conditions as above, but detuned the first resonance laser $0.2 \mathrm{~nm}$ off of the resonance wavelength, from 831.021 to $831.221 \mathrm{~nm}(0.1 \mathrm{~nm}$ change in the second harmonic), while leaving the other lasers unchanged. This removed resonantly ionized $\mathrm{U}$ atoms and gave a direct measurement of the background under the $\mathrm{U}$ atom peaks due to all sources in the instrument. The offresonance spectrum contained $680{ }^{238} \mathrm{U}$ ions per $10^{5}$ laser shots, corresponding to a signal-to-noise ratio of $\sim 20$. Uranium oxides and other molecular species remain however, due to the broad resonances characteristic of molecules. The $\mathrm{U}$ atom-to-oxide ratio was 0.002 for the off-resonance experiment; with an equal number of U-oxide counts $(\sim$ $4 \times 10^{5}$ normalized $\mathrm{UO}_{x}$ counts) as observed in the onresonance experiment.

Finally, we ran a 3-color experiment using laser desorption instead of ion sputtering to generate neutral atoms. Desorption laser power was controlled by a variable neutral density filter using an optical density of $\sim 1.4$, which translated to a laser power of $\sim 5 \mathrm{~mW}$ at the sample surface. The atom-to-oxide ratio improved by more than an order of magnitude compared to ion sputtering, from 0.04 to 0.42 . Running at high desorption laser power produced variable ion signals with intense, sporadic bursts of ions likely resulting from instabilities in the desorption laser, and these data were not able to be interpreted. Running at a lower power and count rate $\left(\sim 8500{ }^{238} \mathrm{U}\right.$ ions per $10^{5}$ laser shots) however, yielded isotope ratios with much larger uncertainties due to the limited number of ions collected (as can been seen in Fig. 5).
None of the 3-color experiments show significant backgrounds in the uranium mass region (Fig. 3, inset). $\mathrm{Ga}^{+}$sputtering and laser desorption, however, differ strongly in the suppression of molecular species. The source of this variation must lie in the coupling of the laser with the sample matrix. It is not yet clear if this effect is specific to desorption from a U-silicate matrix, or if it applies to other U-bearing materials.

\section{2-color RIMS in U-silicate}

While a 3-color laser excitation scheme can be more selective for uranium, use of a 2-color scheme frees up laser resources, and is likely sufficient for many analytical scenarios. We applied a 2-color resonance ionization scheme to explore the sources of non-resonant contributions to the measured $\mathrm{U}$ atomic and molecular signals. In four experiments (one of 14 runs, and three of one run each, Table 1), we collected spectra from 2-color, $\mathrm{Ga}^{+}$sputtering, and then looked at the spectrum generated using only the ion gun, or only the ion gun and one of the two RIMS lasers.

In the initial 2-color experiment, much higher count rates were attained $\left(\sim 75000{ }^{238} \mathrm{U}\right.$ ions per $10^{5}$ laser shots $)$ than was observed in the analogous 3-color experiment. This difference is attributable to using a larger aperture in the primary ion beam ( $1 \mathrm{~mm}$ vs. $300 \mu \mathrm{m})$, which increased the $\mathrm{Ga}^{+}$ current on the sample to $\sim 30 \mathrm{nA}$. The uranium oxide peaks are again prominent, at much higher intensity than the uranium ion signal (Fig. 4), with an atom/molecule ion ratio of $\sim 0.05$, intermediate to that observed in the 3 -color $\mathrm{Ga}^{+}$and laser desorption experiments. No secondary ion background signal was detected in the uranium mass region (Fig. 4, inset) in this experiment.

The secondary ion signal produced by leaving the $\mathrm{Ga}^{+}$ beam on cuprosklodowskite and blocking the lasers is negligible (Fig. 4), with only a few counts in the $\mathrm{UO}_{2}$ mass region $\left(\sim 10^{238} \mathrm{U}\right.$ ions per $10^{5}$ laser shots, Table 1$)$. Admitting the resonance laser $(436.326 \mathrm{~nm})$ while leaving the ionization laser blocked results in 60-fold increase in the ${ }^{238} \mathrm{U}$ ion signal, but produces a larger jump in the U-oxide signal, from $10^{3}$ counts of ${ }^{238} \mathrm{UO}_{2}$ using only the ion gun, to $10^{5}$ counts

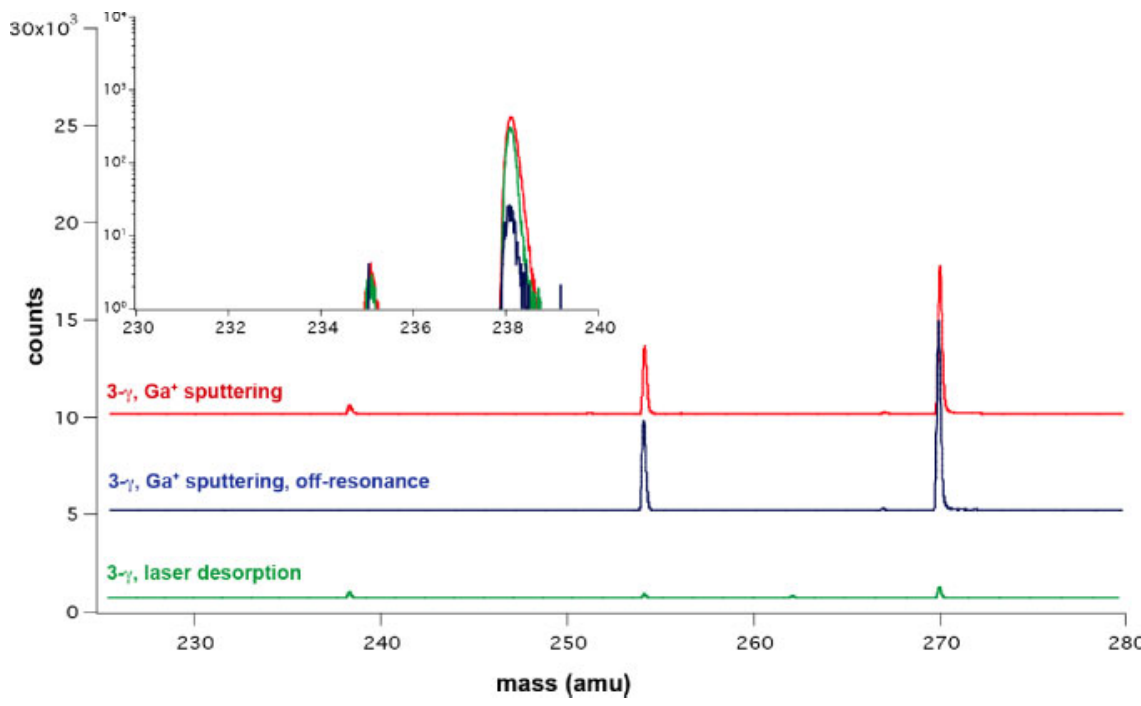

Fig. 3. 3-color U resonance ionization experiments using $\mathrm{Ga}^{+}$sputtering and laser desorption. Experiments are offset in the vertical axis for clarity, illustrating the mass region including the $\mathrm{U}$ atom signal as well as $\mathrm{UO}$ and $\mathrm{UO}_{2}$ oxide signals. The inset shows the $\mathrm{U}$ atom region plotted on a log scale (no offset). 


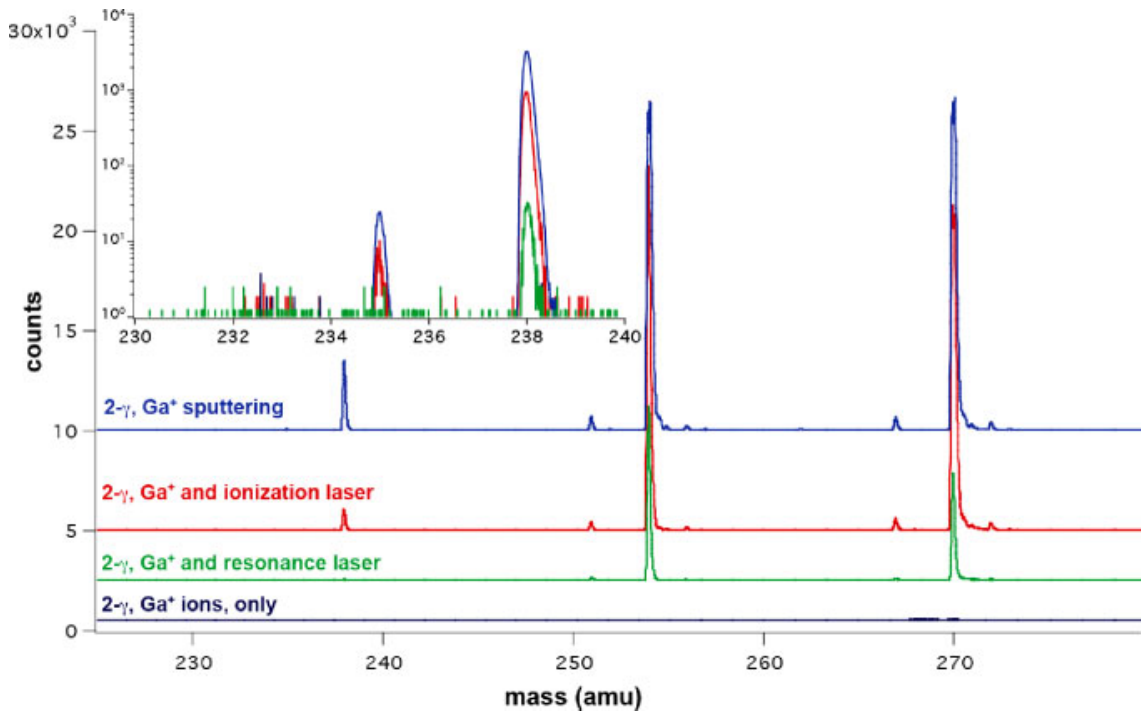

Fig. 4. 2-color U resonance ionization experiments using $\mathrm{Ga}^{+}$sputtering. Experiments are offset in the vertical axis for clarity, illustrating the mass region including the $U$ atom signal as well as $\mathrm{UO}$ and $\mathrm{UO}_{2}$ oxide signals. The inset shows the $\mathrm{U}$ atom region plotted on a log scale (no offset). with the introduction of the resonance laser. Non-resonant photoions, either from one-color, two-photon ionization of neutral uranium or from photo-fragmentation of $\mathrm{UO}_{x}$, are also evident (Fig. 4, inset).

The combination of $\mathrm{Ga}^{+}$sputtering with only the ionization laser $(367.891 \mathrm{~nm})$ results in significantly larger signals in all ion channels, compared to the same experiment using only the resonance laser. There were more than $7 \times 10^{5}$ counts of ${ }^{238} \mathrm{UO}_{2}$, indicating that most of the oxide signal in the 2-color RIMS spectrum is due to one-color, two-photon ionization with the $367.891 \mathrm{~nm}$ laser. In addition, the contribution to the $\mathrm{U}$ atomic ion intensity is large, accounting for $\sim 25 \%$ of the RIMS signal. This is due to a combination of one-color, two-photon ionization of $U$ atoms and photofragmentation of $\mathrm{UO}_{x}$. The latter mechanism likely dominates this signal, since non-resonant ionization of atoms is many orders of magnitude less efficient than resonance ionization [1], and there is no one-color two-photon transition observed for $U$ at this wavelength [12].

The 2-color scheme is potentially less sensitive to isotopic fractionation than the 3 -color scheme since 1) there is only a single resonance transition and hence a single isotope shift to consider, and 2) the isotope shift for the $436.326 \mathrm{~nm}$ transition in the 2-color scheme is smaller than that of the $415.514 \mathrm{~nm}$ transition in the 3-color scheme (4 pm vs. $7 \mathrm{pm}$ ). Fig. 5 shows that this is indeed the case; the ${ }^{235} \mathrm{U} /{ }^{238} \mathrm{U}$ ratio is steady over $14 \times 10^{5}$ laser shots. The measured ${ }^{235} \mathrm{U} /{ }^{238} \mathrm{U}$ ratio was $0.00727 \pm 0.00008(1 \sigma)$ with a $\chi^{2}$ of 1.4 after approximately $1 \mathrm{~h}$ of data acquisition, corresponding to an internal precision of $\sim 1 \%$ (Table 1). Given that $\chi^{2}$ was $\sim 1$ for all the experiments reported in Table 1, the measured precisions are limited only by counting statistics. Simple accumulation of more counts should improve precision. In fact, precision better than $0.5 \%$ has been reported using the 3-color scheme on uranium oxide isotopic standards [3].

The absolute accuracy of the measured ${ }^{235} \mathrm{U} /{ }^{238} \mathrm{U}$ ratio, however, must take into account instrumental fractionation, primarily due to spectroscopic fractionation induced by the isotope shifts. This effect is seen clearly in the difference between the ${ }^{235} \mathrm{U} /{ }^{238} \mathrm{U}$ ratios measured by the 2- and 3color schemes (Table 1). The larger isotope shift for the first resonance transition, plus the additional shift associ- ated with the second resonance transition $(6 \mathrm{pm})$, results in the 3-color scheme having a larger deviation from the natural ${ }^{235} \mathrm{U} /{ }^{238} \mathrm{U}$ ratio of 0.00725 . The 3-color scheme has the advantage of much lower non-resonant (i.e. total) backgrounds than the 2-color scheme and the absolute deviation can be corrected by sample-standard bracketing, as is commonly done in other methods such as secondary ion mass spectrometry.

\section{Useful yields from U-silicate}

The useful yield (the ratio of atoms detected to atoms consumed) was determined for the 2-color scheme using a pulsed $\mathrm{Ga}^{+}$beam to sputter material from the sample. The total U ion signal (73000 ions) was collected over $10^{5}$ laser shots. While hundreds of thousands of atomic and molecular ions were detected, the total amount of material removed during the analysis was too small to measure accurately. Instead, the sputter rate was independently determined by running the ion gun in continuous mode. $\mathrm{A} \mathrm{Ga}^{+}$current of $30 \mathrm{nA}$ was rastered over a $20 \times 20 \mu \mathrm{m}$ area for $160 \mathrm{~s}$ to excavate a crater several microns deep. Three craters were made, in total. The sample was then removed from the instrument, and 3-D crater profiles were measured by optical interferometry (Fig. 6). Crater volumes were determined to be $\sim 3600 \mu \mathrm{m}^{3}$. A normalized sputter rate of $0.3 \mathrm{U}$ atoms for each incident $\mathrm{Ga}^{+}$ion was determined.

The sample uranium concentration $(23.4 \% \mathrm{U})$, measured independently by ICP-MS, was used in combination with the number of $\mathrm{U}$ ions counted over $10^{5}$ laser shots ( $3 \times 10^{7} \mathrm{~ns}$ of analysis time using a $300 \mathrm{~ns} \mathrm{Ga}^{+}$pulse width). The resulting useful yield for uranium from a uranium silicate using $\mathrm{Ga}^{+}$desorption was $5 \times 10^{-5}$ uranium ions detected per atom removed from the sample. We used a literature value of $3.85 \mathrm{~g} / \mathrm{cm}^{3}$ for cuprosklodowskite, which does not account for sample porosity. A lower actual density would have the effect of increasing the calculated useful yield.

A low useful yield measured directly from an oxide, using almost no sample preparation, is not unexpected. Goeringer et al. [13] estimated a useful yield for $\mathrm{U}^{+}$of $\sim 10^{-3}$ 

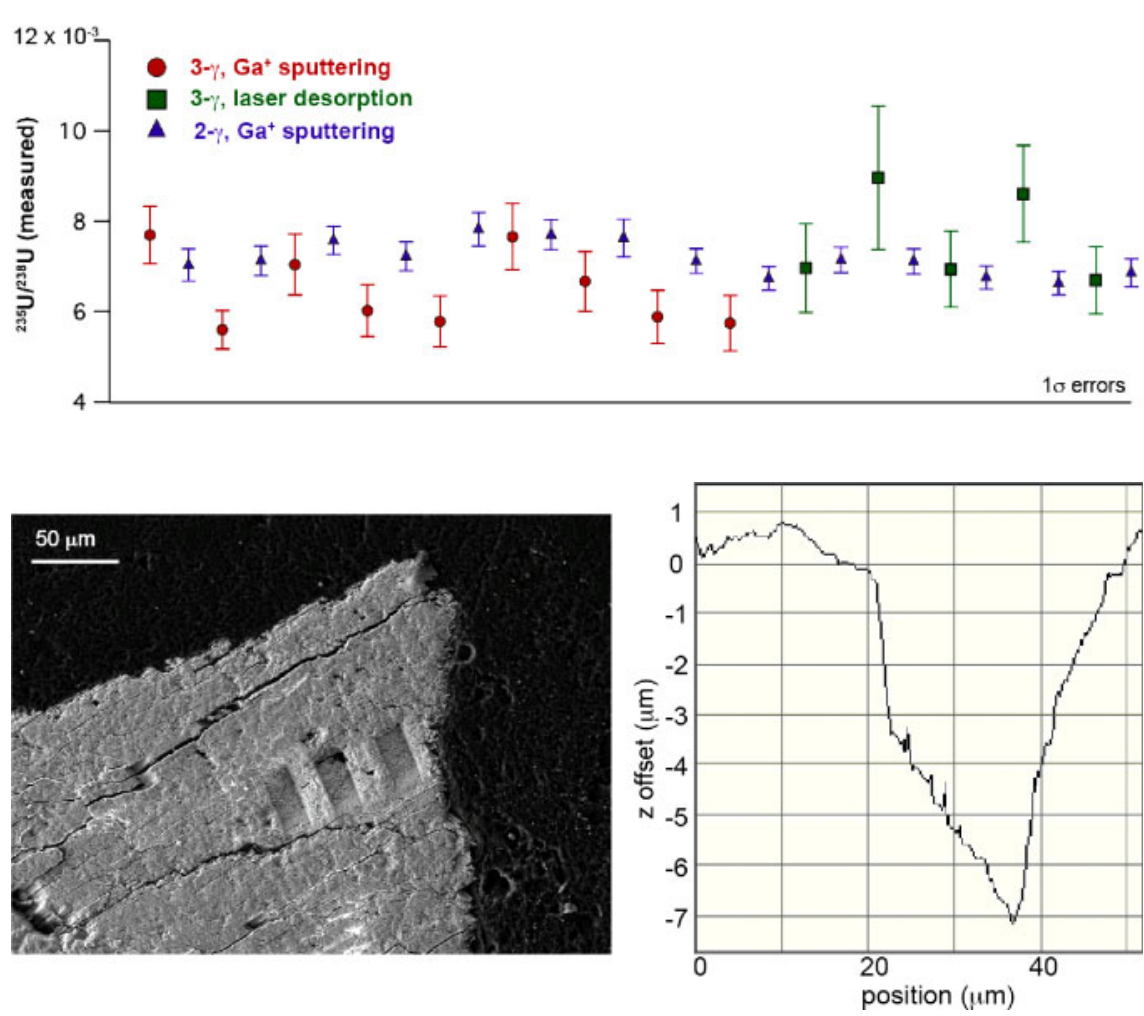

Fig. 5. A summary of ${ }^{235} U /{ }^{238} U$ measurements on cuprosklodowskite by RIMS comparatively illustrating internal analytical precision. Each data point refers to 100000 laser shots. Measured ${ }^{235} \mathrm{U} /{ }^{238} \mathrm{U}$ ratios and errors are given in Table 1.

Fig. 6. A secondary electron image shows three craters created by a $20 \times 20 \mu \mathrm{m} \mathrm{Ga}{ }^{+}$ beam to determine the ion sputtering rate in cuprosklodowskite. Craters were then imaged by optical interferometry. A vertically exaggerated 2-D trace of the crater furthest to the left is shown. Note the sloped shape of the pit caused by the $60^{\circ}$ incidence angle of the $\mathrm{Ga}^{+}$ beam to the sample surface. for RIMS with ion sputtering from uranium metal. Using the atom/oxide ratio in their spectra, their useful yield for $\mathrm{U}^{+}$ from $\mathrm{UO}_{2}$ is approximately 40 times less, or about $2 \times 10^{-5}$. In these experiments, the low useful yield is likely due to the desorbed flux of neutrals from the surface being dominated by molecules. Given the improvement in atom-to-oxide ratios observed using laser desorption on cuprosklodowskite (Table 1), the laser desorption useful yield is likely to be over an order of magnitude greater than that for $\mathrm{Ga}^{+}$sputtering, but has not been directly measured.

\section{Conclusions}

These initial experiments demonstrate that RIMS analyses can be applied directly to environmental samples such as U-silicates for uranium isotope analysis using minimal sample preparation. The method is highly selective for uranium; ionization of other elements is suppressed quantitatively, without the need for purification of the sample. Ionization of molecules (UO and $\mathrm{UO}_{2}$ ), however, is difficult to avoid. Molecular species have broad resonances (the $\mathrm{UO}_{x}$ signals persist as the laser wavelength is scanned over several nanometers), and can be resonantly ionized by the applied RIMS scheme, in addition to being ionized through onecolor, multiphoton processes. These preliminary data suggest that, at least for U-silicate minerals, the production of ionized molecules is strongly dependent on the interaction of the resonance laser with the cloud of desorbed neutral atoms and molecules. This, in turn, implies that there exists a limit as to how well photo-ionization of oxides can be suppressed. This could be problematic if one is seeking to analyze a multi-element sample where an abundant lowmass element forms an oxide isobaric with a heavier element of interest. Therefore the atom-to-oxide ratio must be im- proved. The use of laser desorption appears promising in this regard.

Our low useful yield confirms values suggested by other RIMS experiments on U samples (e.g., [13]), and reflects, in large part, the preferential sputtering of U-oxide molecules (some fraction of which are also ionized) relative to $\mathrm{U}$ atoms. Several approaches, including alternative sample desorption methods, may be used to improve the useful yield. In cuprosklodowskite, for example, laser desorption significantly improves the atom yields relative to $\mathrm{Ga}^{+}$sputtering, by a factor of 10 . This effect may be particular to U-silicate matrices, for example, resulting from a specific coupling of desorption wavelength to the mineral bonds, and needs further exploration.

The use of broadened resonance laser bandwidth in conjunction with an automated feedback system monitoring and adjustment of the resonance and ionization wavelengths stabilizes the $U$ isotope signal within a given experiment. The resulting precision becomes a function of counting statistics in the case of $\mathrm{Ga}^{+}$desorption, or in the case of laserbased desorption, the realization of a stable ion signal. Even with low useful yields, the data reported here show internal precisions as good as $1 \%$. Future studies will seek to optimize analytical conditions for improved suppression of molecular ionization, as well as improving the U useful yield. We will also pursue measurement of uranium isotopes by RIMS from other environmental U-bearing samples including oxide and glass matrices.

Acknowledgment. This work performed under the auspices of the U.S. Department of Energy by Lawrence Livermore National Laboratory under Contract DE-AC52-07NA27344. This work was funded by the Laboratory Directed Research and Development Program at LLNL under project tracking code 10-SI-016, as well as with support from Department of Energy Office of Nonproliferation Research and Development and the Department of Homeland Security. The 
CHARISMA facility at Argonne National Laboratory is funded by the US Department of Energy, Basic Energy Sciences, Division of Materials Science and Engineering under Award No. DEAC02-06CH11357. LLNL-JRNL-422855.

\section{References}

1. Hurst, G. S., Payne, M. G., Kramer, S. D., Young, J. P.: Resonance ionization spectroscopy and one-atom detection. Rev. Mod. Phys. 51, 767-820 (1979).

2. Wendt, K., Trautmann, N.: Recent developments in isotope ratio measurements by resonance ionization mass spectrometry. Int. J. Mass Spectrom. 242, 161-168 (2005).

3. Isselhardt, B. H., Savina, M. R., Knight, K. B., Hutcheon, I. D., Prussin, S. J.: Improving precision in resonance ionization mass spectrometry: influence of laser bandwidth in uranium isotope ratio measurements. Anal. Chem. 83, 2469-2475 (2011).

4. Erdmann, N., Passler, G., Trautmann, N., Wendt, K.: Resonance ionization mass spectrometry for trace analysis of long-lived radionuclides. Radioact. Environ. 11, 331-354 2008.

5. Ziegler, S. L., Bushaw, B. A.: Ultratrace uranium fingerprinting with isotope selective laser ionization spectrometry. Anal. Chem. 80, 6029-6033 2008.

6. Raeder, S., Fies, S., Tomita. H., Wendt, K.: Selective Isotope Determination of Uranium using HR-RIMS. $4^{\text {th }}$ International Con- ference on Laser Probing, American Institute of Physics CP1104, 96-101 (2008).

7. Savina, M. R., Pellin, M. J., Tripa, C. E., Veryvovkin, I. V., Calaway, W. F., Davis, A. M.: Analyzing individual presolar grains with CHARISMA. Geochim. Cosmochim. Acta 67, 3215-3225 (2003).

8. Levine, J., Savina, M. R., Stephan. T., Dauphas, N., Davis, A. M., Knight, K. B., Pellin, M. J.: Resonance ionization mass spectrometry for precise measurements of isotope ratios. Int. J. Mass Spectrom. 288, 36-43 (2009).

9. Ray, A. K., Mago, V. K., Lal B, Rao, P. R. K.: New odd-parity Rydberg and autoionization levels in uranium I. J. Opt. Soc. Am. B 7(2), 145-151 (1990).

10. Schumann, P. G., Wendt, K. D. A., Bushaw, B. A.: High-resolution triple-resonance autoionization of uranium isotopes. Spectrochim. Acta B 60(11), 1401-1411 (2005).

11. Stephan, T., Zehnpfenning, J., Benninghoven, A.: Correction of dead time effects in time-of-flight mass spectrometry. J. Vac. Sci. Technol. A 12(2), 405-410 (1994).

12. Mago, V. K., Ray, A. K., Lal, B., Rao, P. R. K.: Two-step singlecolour photoionization spectroscopy of atomic uranium. Appl. Phys. B 56, 39-42 (1993).

13. Goeringer, D. E., Christie, W. H., Valiga, R. E.: Investigation of matrix effects on the neutral fractions ejected from ionbombarded, uranium-containig solids using resonance ionization mass spectrometry. Anal. Chem. 60, 345-348 (1988). 